\title{
6 \\ Klassifizierung von Komplikationen bei Tätowierungen: Potenzial für neuen Standard
}

Peter Arne Gerber

Klinik für Dermatologie, Universitätsklinikum Düsseldorf

Abstractübersetzung aus Serup J, Sepehri M, Hutton Carlsen K: Classification of tattoo complications in a hospital material of 493 adverse events. Dermatology 2016;232:668-678.

\section{Klassifizierung von Tätowierungskomplikationen in Krankenhausdaten zu 493 unerwünschten Ereignissen}

\section{Schlüsselwörter}

Tätowierung · Komplikation · Allergie · Infektion · Sarkoidose · Pigmente - Tinte · Gesundheitsprobleme · Diagnose .

Klassifikation

\section{Zusammenfassung}

Hintergrund/Ziele: Tätowierungen sind weltweit in Mode. Das klinische Wissen über Komplikationen beruht auf Fallberichten, die im Laufe eines Jahrhunderts gesammelt wurden. Untersuchungen an größeren Kohorten zu Komplikationen und aktuellen Trends liegen nicht vor.

Methoden: Wir analysierten retrospektiv eine Kohorte konsekutiver Patienten mit Tätowierungskomplikationen, die zwischen 2008 und 2015 in der «Tattoo-Klinik» des Universitätskrankenhauses Bispebjerg in Kopenhagen, Dänemark anhand einer Anamnese und der systematischen klinischen Untersuchung diagnostiziert wurden.

Ergebnisse: Insgesamt wurden 493 Tätowierungskomplikationen bei 405 Patienten ausgewertet. In der Summe zeigten 184
(37\%) allergische Reaktionen, 32,2\% mit plaqueförmigen Erhebungen, 3,7\% mit übermäßiger Hyperkeratose und 1,4\% mit Ulzerationen, vor allem bei roten Tätowierungen und Rottönen; 66 (13\%) zeigten papulös-noduläre Reaktionen, vor allem bei schwarzen Tätowierungen (als nicht-allergisch eingestuft) und infolge der Agglomeration von Pigmenten; 53 (11\%) hatten bakterielle Infektionen; bei 46 (9\%) lagen psychosoziale Komplikationen vor; 144 (30\%) fielen in verschiedene diagnostische Einzelkategorien, darunter Lichtempfindlichkeit, Schmerzsyndrom und Lymphopathie. Wir fanden keine Fälle von kutanen oder sonstigen malignen Erkrankungen. Sarkoidose trat vor allem bei schwarzen Tätowierungen auf und stellte im Vergleich zur Basisbevölkerung eine häufige Begleiterkrankung dar, die bei 23 (5\%) Reaktionen vorlag.

Schlussfolgerung: Mit dieser Studie wird ein neues Konzept der Klassifizierung von Tätowierungskomplikationen eingeführt, das auf einfachen Instrumenten wie der Anamnese und objektiven Befunden aus der Histologie fußt. Die Studie spiegelt Komplikationen nach der Verwendung von aktuell gebräuchlichen Tätowierfarben wider, die häufig organische Pigmente enthalten. Die hier eingeführte Klassifizierung wurde der Weltgesundheitsorganisation (WHO) als Vorschlag für die 11. Revision der Internationalen Klassifikation der Krankheiten (ICD) vorgelegt.

(c) 2017 S. Karger GmbH, Freiburg

\section{KARGER}

() 2017 S. Karger GmbH, Freiburg 


\section{Transfer in die Praxis}

\section{Hintergrund}

Das US-amerikanische Marktforschungsunternehmen Harris Interactive berichtet, dass die Prävalenz von Tattoos in den USA im Zeitraum von 2008 bis 2012 um 7\% auf 21\% gestiegen ist (wWW. harrisinteractive.com). Für Deutschland konnte für 2014 eine Querschnittstudie der Gesellschaft für Konsumforschung (GfK) in Kollaboration mit der Ruhr-Universität Bochum eine Prävalenz von 9,1\% ermitteln. Hierbei zeigte sich die höchste Prävalenz mit 22,3\% in der Altersgruppe der 25- bis 34-Jährigen. Auch Serup und Mitarbeiter führen in der hier vorliegenden Arbeit an, dass in Europa ca. 100 Millionen Bürger tätowiert sind - Tendenz steigend. Wer sich ein Tattoo stechen lässt, ist sich zumeist nicht bewusst, dass Tätowierungen auch relevante gesundheitliche Risiken bergen. Diese reichen von Allergien über Infektionen bis hin zu psychosozialen Problemen.

\section{Studienergebnisse}

In ihrer restrospektiven monozentrischen Studie schlagen Serup und Mitarbeiter nun ein neues Konzept zur systematischen Klassifizierung Tattoo-assoziierter Nebenwirkungen vor. Hierzu haben die Autoren 493 Komplikationen ausgewertet, die in einem Zeitraum von 2008 bis 2015 bei 405 verschiedenen Patienten der Tattoo-Klinik in Kopenhagen gesammelt worden waren. Mit der verhältnismäßig hohen Zahl von fast 500 Reaktionen gehört die Studie zu den bis dato umfangreichten publizierten Fallsammlungen. Dies ermöglicht einen guten Überblick über die Frequenz der jeweiligen Komplikationen. Auch die Zuordnung von Kompli- kationen durch einzelne Tätowierungsfarben ist in diesem Kontext sehr hilfreich (Abb. 1).

Die von den Autoren propagierte Klassifikation differenziert nach Level A und B (WHO Diagnostic Classification System) sowie nach diagnostischen Gruppen (Infektion, Entzündung, psychosozial, verschiedene und behandlungsassoziiert). Die Übersicht zeigt, dass in 37\% aller Fälle allergische Reaktionen mit Abstand am häufigsten auftreten, gefolgt von nicht-allergischen Reaktionen (14\%) und bakteriellen Infektionen (11\%). Differenziert nach Farben tauchen Komplikationen häufig vor allem bei Rot (40\%) und Schwarz (35\%) auf. Während bei roten Tätowierungen vielfach lokale allergische Reaktionen auftreten, dominieren bei schwarzen Tätowierungen nicht-allergische Lokalreaktionen und Infektionen. Differenziert nach klinischer Ausprägung traten am häufigsten allergisch induzierte Plaque auf (32,2\%), gefolgt von Licht-induzierten, urtikariellen Reaktionen (18,6\%), nicht-allergischen papulonodulären Läsionen (13\%) und lokoregionären Infektionen (9,9\%). Als häufigste Ursache wurde Auswahl und Menge des eingebrachten Pigments ausgemacht (20,4\%), gefolgt von kontaminierter Farbe (8,2\%). Die häufigsten Komplikationen durch eine Entfernung von Tätowierungen zeigten sich bei Laserbehandlungen (3,6\%). Nichtsdestotrotz gilt die Behandlung mit gütegeschalteten Lasersystemen als unumstrittener Goldstandard in der Tattoo-Entfernung. In 40 Fällen wurden zudem systemische Komplikationen dokumentiert, hierunter bemerkenswerterweise in 23 Fällen $(57,7 \%)$ eine Sarkoidose.
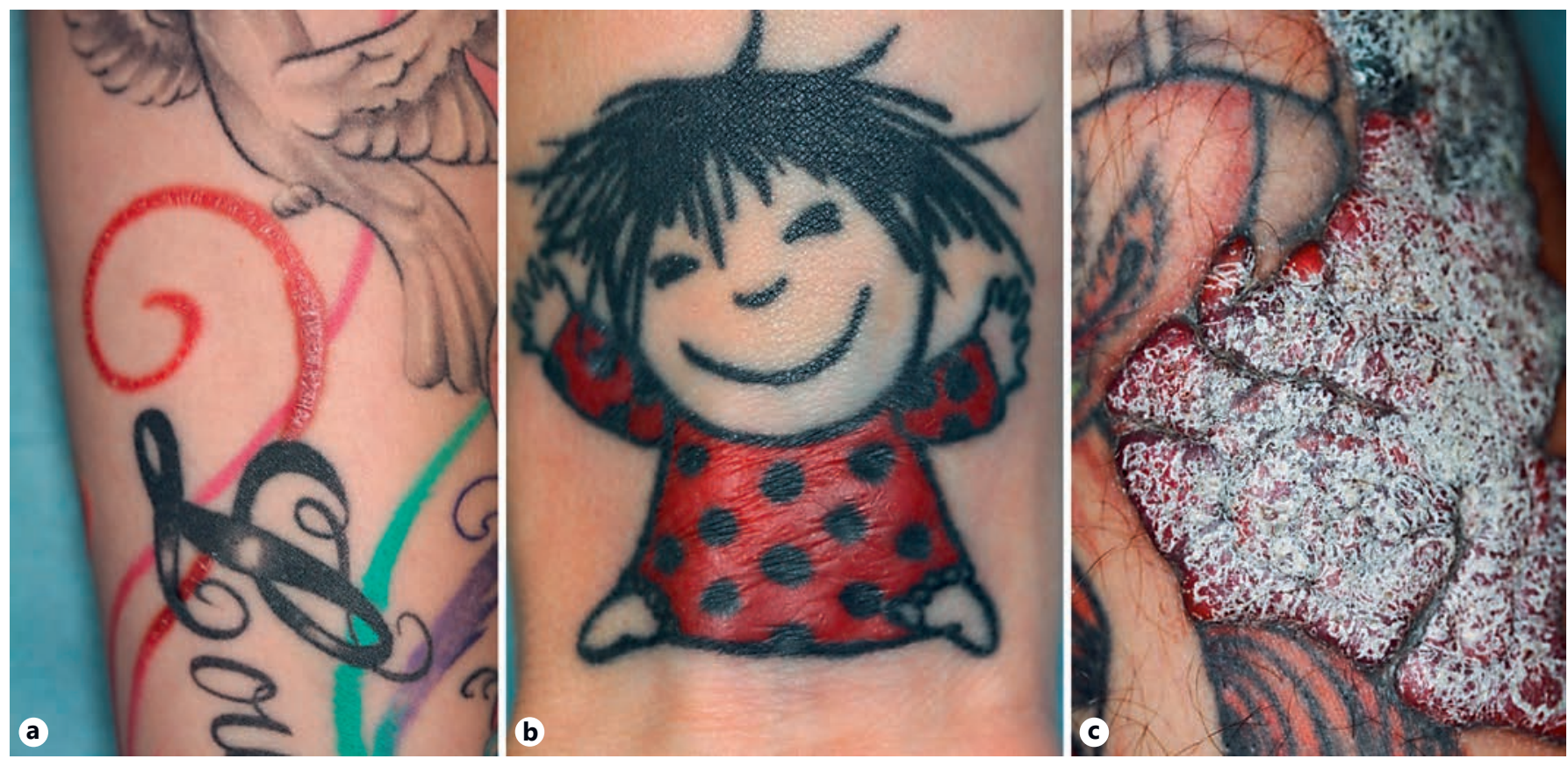

Abb. 1. a Plaque-artige Erhebungen der Haut: gleichmäßige Erhebung mit Abschuppung in jedem Teil des Tattoos in roter Farbe, im Gegensatz zu anderen Farben, die keinerlei Reaktion hervorriefen. b Plaque-artige Erhebungen der Haut: gleichmäßige Erhebung in jedem Teil des Tattoos in roter Farbe ohne Abschuppung, im Gegensatz zu schwarzer Farbe, die keinerlei Reaktion hervorrief. c Exzessive Hyperkeratose: erhebliche Schwellung in den Bereichen des Tattoos in roter Farbe aufgrund von Hautentzündung sowie ausgeprägte hyperkeratotische Reaktion mit epidermaler Hyperplasie, Furchenbildung und begleitender Abschuppung. Man beachte die tiefen Einkerbungen entlang der schwarzen Rahmenlinie des Tattoos bzw. außerhalb. 
Aus ihrem Kollektiv standen den Autoren ferner 259 Hautbiopsien zur Verfügung. Eine histopathologische Auswertung erbrachte in 69\% der Fälle eine Entzündungsreaktion (nicht-granulomatös 69\%; granulomatös 12\%), gefolgt von Sarkoidose (8\%), Pseudolymphom (3\%) und Interface-Dermatitis (3\%).

\section{Fazit für die Praxis}

Die Gruppe um Jørgen Serup gehört zu den international Führenden im Feld der Tattoo-Experten. Das von den Autoren vorgeschlagene System der Klassifikation hat das Potenzial, sich als neuer Standard für die Bewertung Tattoo-assoziierter Komplikationen zu etablieren.

Komplikationen nach Tätowierungen stellen ein in der Bevölkerung unterschätztes gesundheitliches Risiko dar. Dies sollte bei der Beratung von Patienten berücksichtigt werden. So ist einerseits zu empfehlen, die Entscheidung für ein Tattoo auch aus gesundheitlichen Gründen äußerst kritisch abzuwägen. Sofern sich der Patient für eine Tätowierung entscheidet, ist es zum anderen ratsam, insbesondere rote Farben zu meiden, da diese ein überdurchschnittliches Komplikationsrisiko bergen, sowie auf gute Hygienestandards des Tattoo-Studios zu achten.

\section{Disclosure Statement}

Honorare und Reisekostenunterstützung durch Asclepion Laser Technologies.

Kontaktadresse: PD Dr. Peter Arne Gerber, Klinik für Dermatologie, Universitätsklinikum Düsseldorf, Moorenstraße 5, 40225 Düsseldorf, Deutschland, peterarne.gerber@med.uni-duesseldorf.de

A valuable tool for clinical practice

\section{Diagnosis and Therapy of Tattoo Complications}

\section{With Atlas of Illustrative Cases \\ Editors: Jørgen Serup, Wolfgang Bäumler}

Tattooing breaches the skin and can, therefore, cause a variety of complications. This book covers the full spectrum of issues clinical practitioners may encounter when treating affected patients. Introductory chapters include educational information on methods for tattooing, types of tattoos, tattoo inks, and tattoo ink toxicology. The focus is on the diagnosis and classification of tattoo complications. In this regard, a comprehensive atlas of acute and chronic complications serves as a valuable tool. Further chapters summarize available therapies, their rationale, and indication.

Current Problems in Dermatology, Vol. 52

\section{Diagnosis and Therapy of Tattoo Complications}

With Atlas of Illustrative Cases

Editors: Serup, J. (Copenhagen); Bäumler, W. (Regensburg)

VIII + 234 p., 242 fig., 239 in color, 8 tab., 2017

CHF 168.00/EUR 157.00/USD 198.00 (hard cover or online*)

CHF 202.00 / EUR 188.00 / USD 238.00 (online**)

ISBN 978-3-318-05977-9 / e-ISBN 978-3-318-05978-6 *online prices for personal customers

**online prices for institutional purchase

Prices subject to change, VAT not included

EUR price for eurozone countries,

USD price for USA and Latin America only

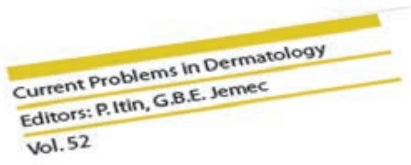

Vol. 52 